\title{
CENTRAL EXTENSIONS AND RATIONAL QUADRATIC FORMS
}

\author{
YOSHIOMI FURUTA AND TOMIO KUBOTA
}

\section{Introduction}

The purpose of this paper is to characterize by means of simple quadratic forms the set of rational primes that are decomposed completely in a non-abelian central extension which is abelian over a quadratic field. More precisely, let $L=$ $\mathbf{Q}\left(\sqrt{d_{1}}, \sqrt{d_{2}}\right)$ be a bicyclic biquadratic field, and let $K=\mathbf{Q}\left(\sqrt{d_{1} d_{2}}\right)$. Denote by $S_{K}(\tilde{m})$ the ray class field $\bmod m$ of $K$ in narrow sense for a large rational integer $m$. Let $L_{m}^{*}$ be the maximal abelian extension over $\mathbf{Q}$ contained in $S_{K}(\tilde{m})$ and $\hat{L}_{m}$ be the maximal extension contained in $S_{K}(\tilde{m})$ such that $\operatorname{Gal}\left(\hat{L}_{m} / L\right)$ is contained in the center of $\operatorname{Gal}\left(\hat{L}_{m} / \mathbf{Q}\right)$. Then we shall show in Theorem 2.1 that any rational prime $p$ not dividing $d_{1} d_{2} m$ is decomposed completely in $L_{m}^{*} / \mathbf{Q}$ if and only if $p$ is representable by rational integers $x$ and $y$ such that $x \equiv 1$ and $y \equiv 0 \bmod m$ as follows

$$
p=\frac{a x^{2}+b x y+c y^{2}}{a}
$$

where $a, b, c$ are rational integers such that $b^{2}-4 a c$ is equal to the discriminant of $K$ and $(a)$ is a norm of a representative of the ray class group of $K \bmod m$. Moreover $p$ is decomposed completely in $\hat{L}_{m} / L_{m}^{*}$ if and only if $\left(\frac{d_{1}}{a}\right)=1$.

\section{§1. Central extensions with respect to quadratic fields}

Let $d_{1}$ and $d_{2}$ be square free integers and let $d_{1} d_{2}=d_{0} d^{2}$, where $d_{0}$ is square free and $d_{0} \neq 1$. Let $K=\mathbf{Q}\left(\sqrt{d}_{0}\right), L=\mathbf{Q}\left(\sqrt{d}_{1}, \sqrt{d}_{2}\right)$ and $D$ be the discriminant of $K$. For a rational integer $m$, denote by $\varsigma_{K}(\tilde{m})$ the ray class $\bmod m$ of $K$ in narrow sense, and by $S_{K}(\tilde{m})$ the ray class field $\bmod m$ of $K$ in narrow sense.

Let $m$ be a rational integer such that $L$ is contained in $S_{K}(\tilde{m})$. Let $L_{m}^{*}$ and $\hat{L}_{m}$

Received June 16,1992 
be the genus field and the central class field of $L / \mathbf{Q}$ with respect to $S_{K}(\tilde{m})$. They are by definition, the maximal subfields of $S_{K}(\tilde{m})$ such that $L_{m}^{*}$ is abelian over $\mathbf{Q}$ and $\operatorname{Gal}\left(\hat{L}_{m} / L\right)$ is contained in the center of $\operatorname{Gal}\left(\hat{L}_{m} / \mathbf{Q}\right)$.

We have $\left[\hat{L}_{m}: L_{m}^{*}\right] \leq 2$ in general, and $\left[\hat{L}_{m}: L_{m}^{*}\right]=2$ when $m$ is large enough, for instance $m$ is a multiple of $4 d d_{0}$. More precisely, let $m_{1}$ be the product of all odd rational primes $q$ such that $q$ divides $d_{0}$ and satisfies both $\left(d_{1} / q\right) \neq 1$ and $\left(d_{2} / q\right) \neq 1$. Define $m_{0}$ by

(1.1) $m_{0}=\left\{\begin{aligned} d m_{1} & \text { when } d_{1} \equiv d_{2} \equiv 1 \bmod 4, \\ d m_{1} & \text { when } d_{i} \equiv 1 \bmod 8, \quad \text { and } d_{j} \not \equiv 1 \bmod 4, \\ 2 d m_{1} & \text { when } d_{i} \equiv 5 \bmod 8, \quad \text { and } d_{j} \not \equiv 1 \bmod 4, \\ 4 d m_{1} & \text { otherwise, }\end{aligned}\right.$

where $i, j=1$ or 2 and $i \neq j$. Then [2, Proposition 3.4] implies $\left[\hat{L}_{m}: L_{m}^{*}\right]=2$ when $m$ is a multiple of $m_{0}$.

Now let $K_{\#}^{*}$ be the genus field of $K$ in absolute sense, and let $\mathbf{Q}(\tilde{m})$ be the ray class field $\bmod m$ of $\mathbf{Q}$ in narrow sense. Let $K_{m}^{*}$ be the genus field of $K / \mathbf{Q}$ with respect to the ray class field $\bmod m$ of $K$ in narrow sense. Then $K_{m}^{*}=L_{m}^{*}$ by the definition, and we have

$$
L_{m}^{*}=K_{\#}^{*} \mathbf{Q}(\tilde{m})
$$

by [2, Theorem 4.3$]$. Thus the genus field $L_{m}^{*}$ is given explicitly as follows

$$
L_{m}^{*}=\Pi \mathbf{Q}\left(\sqrt{q^{*}}\right) \cdot \mathbf{Q}\left(\zeta_{m}\right)
$$

where $q$ runs over all rational primes dividing $d_{0}$, and $q^{*}$ are prime discriminants, i.e., $D=\Pi q^{*}$ by $q^{*}=(-1)^{(q-1) / 2} q,-4$, or \pm 8 .

For the later use, let $\mathfrak{S}_{K}^{\prime}(m)$ be the group of principal ideals $(\alpha)$ of $K$ such that $\alpha \equiv 1 \bmod m$ and $\mathbf{N}_{K / \mathbf{Q}} \alpha>0$, and $S_{K}^{\prime}(m)$ be the class field of $K$ corresponding to $\mathfrak{S}_{K}^{\prime}(m)$. Let $L_{m}^{* \prime}$ and $\hat{L}_{m}^{\prime}$ be the genus field and the central class field of $L / \mathbf{Q}$ with respect to $S_{K}^{\prime}(m)$. Then we can show that

$$
L_{m}^{* \prime}=L_{m}^{*}
$$

and

$$
\hat{L}_{m}^{\prime}=\hat{L}_{m}
$$

as follows.

The ideal group of $K$ corresponding to $\mathbf{Q}(\tilde{m})$ is the group of ideals $\mathfrak{a}$ of $K$ such that $|\mathbf{N a}| \equiv 1 \bmod m$. This group contains $\widetilde{S}_{K}^{\prime}(m)$ and clearly $S_{K}^{\prime}(m) \supset K_{\#}^{*}$. Hence $S_{K}^{\prime}(m)$ contains $L_{m}^{*}=K_{\#}^{*} \mathbf{Q}(\tilde{m})$. This implies (1.3) since $S_{K}(\tilde{m})$ contains 
$S_{K}^{\prime}(m)$.

In order to show (1.4), let $\sigma$ be the non-trivial element of $\mathrm{Gal}(K / \mathbf{Q})$, and denote by $\Omega$ resp. $\Omega^{\prime}$ the group of ideals $\mathfrak{a}$ of $K$ such that $\mathfrak{a}^{\sigma} \equiv \mathfrak{a} \bmod \Im_{K}(\tilde{m})$ resp. $\bmod \mathfrak{S}_{K}^{\prime}(m)$. Then by [1, Proposition 5.1$]$ we have

$$
\operatorname{Gal}\left(\hat{L}_{m} / L_{m}^{*}\right) \cong I_{K} / \mathfrak{H}(L / K) \mathfrak{\Re}
$$

and

$$
\operatorname{Gal}\left(\hat{L}_{m}^{\prime} / L_{m}^{* \prime}\right) \cong I_{K} / \mathfrak{H}(L / K) \mathfrak{R}^{\prime}
$$

where $I_{K}$ is the group of ideals of $K$ prime to $m$ and $\mathfrak{S}(L / K)$ is the subgroup of $I_{K}$ corresponding to $L$ by class field theory. Let $\alpha=1+4 \sqrt{D} m$. Then $(\alpha) \in$ $\mathfrak{H}(L / K)$, because

$$
\left(\frac{d_{i}}{N_{K / \mathbf{Q}} \alpha}\right)=\left(\frac{N_{K / \mathbf{Q}} \alpha}{d_{i}}\right)=1
$$

for $i=1,2$, since $N_{K / \mathbf{Q}} \alpha \equiv 1 \bmod 8$. When $\mathfrak{S}_{K}^{\prime}(m) \neq \mathfrak{S}_{K}(\tilde{m})$, the non-trivial class of $\mathfrak{S}_{K}^{\prime}(m) / \mathfrak{S}_{K}(\tilde{m})$ is represented by $1-m$, and $1-m=\alpha^{1-\sigma} \alpha_{1}$, where $\alpha_{1}=$ $\alpha^{\sigma-1}(1-m)$, which is contained in $\mathfrak{S}_{K}(\tilde{\mathfrak{m}})$. Thus for any element $(\gamma)$ of $\mathfrak{S}_{K}^{\prime}(m)$, we have $(\gamma)=(\alpha)^{1-\sigma}\left(\gamma_{1}\right)$, where $\left(\gamma_{1}\right) \in \mathfrak{S}_{K}(\tilde{m})$. Now let a be any element of $\mathfrak{\Omega}^{\prime}$. Then there is $\gamma$ of $K^{\times}$such that $\mathfrak{a}^{\sigma}=\mathfrak{a}(\gamma),(\gamma) \in \mathfrak{S}_{K}^{\prime}(m)$. The above argument implies $(\mathfrak{a}(\alpha))^{\sigma}=\mathfrak{a}(\alpha)\left(\gamma_{1}\right)$, that is $\mathfrak{a}(\alpha) \in \mathfrak{\Omega}$. Hence $\mathfrak{a} \in(\alpha)^{-1} \mathfrak{\Re} \subset \mathfrak{H}(L / K) \mathfrak{R}$. Therefore $\left[\hat{L}_{m}^{\prime}: L_{m}^{*}\right]=2$ if and only if $\left[\hat{L}_{m}: L_{m}^{*}\right]=2$ by (1.3), (1.5) and (1.6). This implies further (1.4) because of definition of central extensions and $\mathfrak{S}_{K}(\tilde{m}) \subset \mathfrak{S}_{K}^{\prime}(m)$.

\section{§2. Decomposition of primes}

Notation being as in the preceding section, let $\mathfrak{B}$ be an ideal of $L_{m}^{*}=L_{m}^{*}$, prime to $m$. Then it follows from the definition of the genus field that there exists an ideal $a$ of $K$ such that

$$
\mathfrak{a}^{\sigma-1} \equiv \mathbf{N}_{L_{m}^{*} / K} \mathfrak{B} \bmod \mathfrak{S}_{K}^{\prime}(m)
$$

Let $\mathfrak{b}=N_{L_{m}^{*} / K} \mathfrak{B}$ and $(a)=N_{K / \mathbf{Q}} \mathfrak{a}$. Suppose that no prime divisor of $\mathfrak{a}$ ramified in $L$. Then by [2, Proposition 1.5] exchanged the notation $a$ and $b$, we have the following relation of Artin symbols:

$$
\left(\frac{\hat{L}_{m} / L_{m}^{*}}{\mathfrak{B}}\right)=\left(\frac{\hat{L}_{m} / K}{\mathfrak{b}}\right)=\left(\frac{L / K}{\mathfrak{a}}\right)=\left(\frac{d_{1}}{a}\right)=\left(\frac{d_{2}}{a}\right) .
$$


Let $C_{m}^{\prime}(\mathfrak{a})$ be the class of ideals of $K \bmod \widetilde{S}_{K}^{\prime}(m)$ which contains $\mathfrak{a}$, and let $\mathfrak{N}\left(C_{m}^{\prime}(\mathfrak{a})\right)$ be the set of norms of "integral" ideals contained in $C_{m}^{\prime}(\mathfrak{a})$. Then any rational prime of $\mathfrak{\Re}\left(C_{m}^{\prime}\left(\mathrm{a}^{1-\sigma}\right)\right)$ not dividing $m$ is decomposed completely in $L_{m}^{*}=$ $L_{m}^{* \prime}$. It is further decomposed completely in $\hat{L}_{m}$ when $\left(\frac{d_{1}}{a}\right)=1$ by $(2.2)$, where $(a)=N_{K / \mathbf{Q}^{\mathfrak{a}}}$.

Let us call a rational integer $D$ a discriminant integer when there is a quadratic field whose discriminant is equal to $D$. For a discriminant integer $D$ and a rational integer $m$, denote by $A(D, m)$ the set of rational integers $a$ satisfying the following condition:

$$
\left\{\begin{array}{l}
a \text { is square free, and g.c.d. }(a, m)=1 . \\
\left(\frac{D}{q}\right)=1 \text { for all odd prime factors } q \text { of } a . \\
a \text { is odd, if } D \neq \equiv 1 \bmod 8 .
\end{array}\right.
$$

Note that $a \in A(D, m)$ implies that $(a)$ is a norm of an integral ideal of $K$ prime to $m$.

For a rational integer $a$ in $A(D, m)$, choose a primitive integral form $a x^{2}+b x y+c y^{2}$ with discriminant $D$, and define $H(D, m, a)$ by

$$
H(D, m, a)=\left\{\frac{a x^{2}+b x y+c y^{2}}{a} \in \mathbf{Z} ; x \equiv 1, y \equiv 0 \bmod m\right\} .
$$

Note that $H(D, m, a)$ is independent of the choice of $b, c$, because if $b_{1}^{2}-$ $4 a c_{1}=D$ too, then $b=b_{1}+2 a t$ by $t \in \mathbf{Z}$ and we have

$$
{ }^{t} U\left[\begin{array}{cc}
a_{1} & b_{1} / 2 \\
b_{1} / 2 & c_{1}
\end{array}\right] U=\left[\begin{array}{cc}
a & b / 2 \\
b / 2 & c
\end{array}\right]
$$

by $U=\left[\begin{array}{ll}1 & t \\ 0 & 1\end{array}\right]$

THEOREM 2.1. Let $L=\mathbf{Q}\left(\sqrt{d_{1}}, \sqrt{d_{2}}\right)$, where $d_{1}$ and $d_{2}$ are distinct square free integers and $d_{1} d_{2}=d_{0} d^{2}$ by a square free integer $d_{0}$. Let $m$ be an integer divisible by $m_{0}$ defined in (1.1). Let $L_{m}^{*}$ and $\hat{L}_{m}$ be the genus field and the central class field of $L / \mathbf{Q}$ with respect to the ray class field $\bmod m$ of $K$. Let $p$ be a rational prime not dividing $d_{1} d_{2} m$. Then $p$ is decomposed completely in $L_{m}^{*} / \mathbf{Q}$ if and only if $p$ is contained in $H(D, m, a)$ for some rational integer $a$ of $A(D, m)$. It is further decomposed completely in $\hat{L}_{m} / L_{m}^{*}$ if and only if $\left(\frac{d_{1}}{a}\right)=1$. 
Proof. By (2.2) and (2.1), it is enough to show that

$$
p \in H(D, m, a) \Leftrightarrow p \in \mathfrak{R}\left(C_{m}^{\prime}\left(a^{1-\sigma}\right)\right),
$$

where $\mathfrak{a}$ is an integral ideal of $K$ and $(a)=\mathbf{N}_{K / \mathbf{Q}} \mathfrak{a}$.

Suppose that $p \in \mathfrak{N}\left(C_{m}^{\prime}\left(\mathfrak{a}^{1-\sigma}\right)\right)$. Then there are a prime ideal $\mathfrak{p}$ dividing $p$ and an element $\alpha$ of $K$ such that $\mathfrak{p}=(\alpha) \mathfrak{a}^{1-\sigma}, \alpha \equiv 1 \bmod m$ and $\alpha \alpha^{\sigma}>0$. We can assume that $a$ contains no rational divisor. Then we have $\mathfrak{a}^{-1} \cap \mathbf{Q}=\mathbf{Z}$, since any multiple divisor of $a^{-1}$ is rational only if it is integral. Hence we can choose a $\mathbf{Z}$-basis of $a^{-1}$ in the form $\{1, \omega\}$ by an element $\omega$ of $K$. Let $\alpha=x+\omega y$, where $x, y \in \mathbf{Z}$. Then

$$
p=\alpha \alpha^{\sigma}=(x+\omega y)\left(x+\omega^{\sigma} y\right) .
$$

Let $(a)=N_{K / \mathbf{Q}}$ a. Then $a \in A(D, m)$. Since the ideal divisor (Inhalt) of the polynomial $x+\omega y$ is equal to $\mathrm{a}^{-1}$, the rational quadratic form $a(x+\omega y)(x+$ $\left.\omega^{\sigma} y\right)$ must be primitive. Denote this form by $a x^{2}+b x y+c y^{2}$. Then $D=b^{2}-$ $4 a c$ and we have

$$
p=\frac{a x^{2}+b x y+c y^{2}}{a}
$$

where $x \equiv 1, y \equiv 0 \bmod m$, since $\alpha \equiv 1 \bmod m$ and g.c.d. $(a, m)=1$.

Conversely suppose that $p \in H(D, m, a)$, where $D=b^{2}-4 a c$ and $a \in$ $A(D, m)$. Let $\alpha=x+\omega y$, where $\omega=\left(b+\sqrt{b^{2}-4 a c}\right) / 2 a \in K$. Then $\alpha \in$ $S_{K}^{\prime}(m)$ and $p=N_{K / \mathbf{Q}} \alpha$. Compare the decomposition of the both sides to prime ideals. Then we see that there exists a prime ideal $\mathfrak{p}$ and an integral ideal $\mathfrak{a}$ of $K$ such that $(p)=N_{K / \mathbf{Q}} \mathfrak{p}, \mathfrak{p}=(\alpha) \mathfrak{a}^{1-\sigma}$ and $a=N_{K / \mathbf{Q}} \mathfrak{a}$. This completes the proof.

Remark 2.1. For a given pair of integers $d_{0}$ and $m$, the number of distinct sets $\mathfrak{N}\left(C_{m}^{\prime}(\mathfrak{a})\right)$ is not exceed the number of the classes $\bmod \widetilde{S}_{K}^{\prime}(m)$. Hence the set of rational primes decomposed completely in $\hat{L}_{m} / \mathbf{Q}$ coincides with the union of rational primes contained in $H(D, m, a)$ by a finite number of rational integers $a$ satisfying the condition (2.3) and $\left(\frac{d_{1}}{a}\right)=1$.

Remark 2.2. The set of rational primes decomposed completely in $\hat{L}_{m} / \mathbf{Q}$ coincides also with the set of primes $p$ such that $\left[d_{1}, d_{2}, p\right]=1$, where the symbol is defined in [2]. On a treatment by means of this symbol in a restricted case, see [4, Proposition 3.1]. 


\section{REFERENCES}

[1] Y. Furuta, Note on class number factors and prime decompositions, Nagoya Math. J., 66 (1977), $167-182$.

[2] - A prime decomposition symbol for a non-abelian central extension which is abelian over a bicyclic biquadratic field, Nagoya Math. J., 79 (1980), 79-109.

[3] - A norm residue map for central extensions of an algebraic number field, Nagoya Math. J., 93 (1984), 61-69.

[4] - Gauss's ternary form reduction and its application to a prime decomposition symbol, Nagoya Math. J., 98 (1985), 77-86.

Y. Furuta

Department of Mathematics

Faculty of Science

Kanazawa University

Kanazawa 920

Japan

T. Kubota

Department of Mathematics

School of Science

Nagoya University

Chikusa-ku, Nagoya 464-01

Japan

current address:

Department of Mathematics

Meijo University

Nagoya, 468

Japan 